Originalien

Z Gerontol Geriat 2021 · 54:255-263 https://doi.org/10.1007/s00391-020-01730-5 Eingegangen: 10. März 2020

Angenommen: 7. April 2020

Online publiziert: 29. April 2020

(c) Der/die Autor(en) 2020

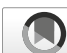

Check for
updates

Anna Greta Barbe' - Sabine Spiritus ${ }^{1} \cdot$ Anna Hagemeier ${ }^{2}$ Michael J. Noack . Gabriele Röhrig ${ }^{3}$

'Fachbereich Parodontologie, AG Seniorenzahnmedizin, Poliklinik für Zahnerhaltung u. Parodontologie, Uniklinik Köln, Köln, Deutschland

${ }^{2}$ Institute of Medical Statistics and Computational Biology, Medical Faculty, University of Cologne, Köln, Deutschland

${ }^{3}$ Zentrum für spezialisierte geriatrische Diagnostik, MVZ Medicum Köln Ost, Köln, Deutschland

\title{
Erfassung der Mundgesundheit von ambulant betreuten Senioren durch Hausärzte
}

\section{Entwicklung und Validierung des geriatrischen ambulanten Mundgesundheits-Screenings}

Assessments erfasst wird [9]. Halitosis gilt seit Jahren als Epiphänomen für eine Reihe mundgesundheitsbezogener Probleme, insbesondere bei geriatrischen Patienten [10]. Die Multikausalität oraler Erkrankungen einerseits und die multidimensionalen Folgen andererseits, die von eingeschränkter Nahrungsaufnahme mit sekundärer Malnutrition über enorale Schmerzen und Kaubeschwerden bis hin zu Aspirationspneumonien mit erhöhtem Mortalitätsrisiko reichen, haben die Diskussion angeregt, eingeschränkte Mundgesundheit im Alter als eigenständiges geriatrisches Syndrom zu betrachten [11]. Dabei gibt es eine überzeugende Studienlage darüber, dass durch eine angemessene Mundpflege bis ins hohe Alter die Gesundheit sowohl von Mund als auch Gesamtorganismus positiv beeinflusst werden kann [12]. Es hat sich gezeigt, dass v. a. ambulant betreute Senioren mit zunehmendem Pflegebedarf den Anschluss an die zahnmedizinische Versorgung kontinuierlich verlieren [13]. Folge dieser Entwicklung ist eine eher problem- als präventionsorientierte Inanspruchnahme zahnärztlicher Dienste. Die weit verbreitete Empfehlung, alle 6 Monate einen Zahnarzt aufzusuchen, scheint für diese vulnerable Patientenpopulation kaum umsetzbar, da einerseits
Mobilitätseinschränkungen ein Hindernis darstellen, andererseits aber auch die Möglichkeiten einer flächendeckend aufsuchenden mobilen zahnmedizinischen Versorgung zum jetzigen Zeitpunkt noch nicht ausreichend gegeben sind. Im Gegensatz zum Zahnarzt werden Hausärzte auch von hochaltrigen Patienten regelmäßig konsultiert und könnten daher als Schlüsselfaktor auch für auftretende zahnmedizinische Gesundheitsprobleme angesehen werden. Es gibt bisher im deutschsprachigen Raum weder geeignete validierte Erfassungsbogen von Mundgesundheit bei älteren Patienten im hausärztlichen Setting, noch gibt es einheitliche Empfehlungen zur Vorgehensweise, wie Mundgesundheit bei älteren Patienten von hausärztlicher Seite miterfasst werden könnte. In der Literatur ist das bekannteste Instrument zur Beurteilung der Mundgesundheit die Brief Oral Health Status Examination (BOHSE), die hauptsächlich für Pflegekräfte in Pflegeheimen entwickelt, validiert und angewendet wurde und sowohl Befragung als auch eine kurze orale Untersuchung beinhaltet [14]. Ebenso sind Oral Health Assessment Tool (OHAT) [15] und Mini Dental Assessment [16] Instrumente, die entweder nach einer zusätzlichen Person oder ei- auf Kau- und Schluckfunktion nicht routinemäßig im Rahmen geriatrischer 
Tab. 1 Beschreibung der relevanten Mundgesundheitprobleme von Senioren, die korrespondierenden zahnmedizinischen Untersuchungsparameter sowie die hierzu entwickelte repräsentative Frage im GAMS

\begin{tabular}{|c|c|c|}
\hline & Mundgesundheitsproblem & Zahnmedizinischer Untersuchungsparameter \\
\hline Haben Sie Gesundheitsprobleme im Mundbereich? & Subjektive Gesamtmundgesundheit & - \\
\hline $\begin{array}{l}\text { 1. Leiden Sie an Missempfindungen wie Brennen } \\
\text { oder pelzigem Gefühl im Mund? }\end{array}$ & Mundschleimhautprobleme & $\begin{array}{l}\text { Druckstellen } \\
\text { Einbisse } \\
\text { Candidiasis } \\
\text { Rhagaden }\end{array}$ \\
\hline $\begin{array}{l}\text { 2. Haben Sie Schmerzen oder andere Beschwerden } \\
\text { im Bereich von Mund und Zähnen? }\end{array}$ & $\begin{array}{l}\text { Kariöse Läsionen, Kronenfrakturen, } \\
\text { zerstörte Zähne }\end{array}$ & $\begin{array}{l}\text { Kariös } \\
\text { Zerstört } \\
\text { Druckstellen } \\
\text { Einbisse } \\
\text { Candidiasis } \\
\text { Rhagaden }\end{array}$ \\
\hline $\begin{array}{l}\text { 3. Haben Sie Zahnfleischbluten beim Putzen, beim } \\
\text { Essen oder auch spontan? }\end{array}$ & $\begin{array}{l}\text { Gingivitis, Parodontitis, mangelhafte } \\
\text { Mundhygiene }\end{array}$ & $\begin{array}{l}\text { Approximaler Plaque-Index } \\
\text { Sulcus-Blutungsindex } \\
\text { Gesamt-PSI } \\
\text { Höchster PSI-Wert }\end{array}$ \\
\hline $\begin{array}{l}\text { 4. Haben Sie Schwierigkeiten beim Zerkauen und } \\
\text { beim Schlucken von Möhren und Äpfeln? }\end{array}$ & Reduzierte Kaufunktion & $\begin{array}{l}\text { Reduzierte Zahnzahl } \\
\text { Kariös } \\
\text { Zerstört } \\
\text { Ersetzt } \\
\text { Xerostomie } \\
\text { Unstimulierte Speichelfließrate } \\
\text { Stimulierte Speichelfließrate }\end{array}$ \\
\hline $\begin{array}{l}\text { 5. Ist Ihnen selbst ein unangenehmer Geruch aus } \\
\text { dem Mund aufgefallen, oder hat Sie jemand darauf } \\
\text { angesprochen? }\end{array}$ & Halitosis & Organoleptischer Index (0-3) \\
\hline $\begin{array}{l}\text { Haben Sie öfter das Gefühl, dass Ihr Mund trocken } \\
\text { ist? }\end{array}$ & Mundtrockenheit & $\begin{array}{l}\text { Xerostomie } \\
\text { Unstimulierte Speichelfließrate } \\
\text { Stimulierte Speichelfließrate }\end{array}$ \\
\hline 6. Haben Sie Voll- oder Teilprothesen? & $\begin{array}{l}\text { Vorhandene Prothetik mit regelmäßiger } \\
\text { Notwendigkeit einer zahnärztlichen } \\
\text { Kontrolle }\end{array}$ & Prävalenz prothetischer Versorgung \\
\hline
\end{tabular}

ner Form der objektiven Untersuchung verlangen. Die Erfahrung zeigt, dass die Untersuchung des Mundes für Nichtzahnärzte ein großes Hindernis darstellt. Sicherlich am erfolgversprechendsten hinsichtlich einer Verbesserung dieser Situation wären Modelle, die neben solchen Erhebungsinstrumenten auch relevante Aspekte wie die Schaffung von Strukturen verbesserter integrierter Versorgung oder entsprechende Anpassung von Ausbildungsinhalten zum Ziel haben.

Die Arbeitsgruppe um Gil-Montoya konnte in ihrem nichtsystematischen Übersichtsartikel nicht nur die enge Verknüpfung der häufigsten Mundgesundheitsprobleme mit geriatrischen Syndromen detailliert aufzeigen, sondern auch, daraus resultierend, den dringenden Bedarf nach einem ganzheitlichen Diagnostikansatz („comprehensive geriatric assessment", CGA) unterstreichen, der neben biopsychosozialen und funktionellen Aspekten auch den oralen Gesundheitsstatus integriert [13]. Dieser Bedarf wird umso dringender hinsichtlich der wachsenden Datenlage über Präventionseffekte von effektiver Mundhygiene: So konnte durch Anwendung chlorhexidinhaltiger Mundpflegeprodukte das Risiko für Pneumonien bei Langzeitbeatmungen und krankenhausassoziierte Pneumonien („hospital-acquired pneumonias“) reduziert werden [17].

Während sich die theoretischen Kenntnisse über die enge Verknüpfung zwischen Mundgesundheit und systemischen Gesundheitsproblemen sowie geriatrischen Syndromen bei den meisten Akteuren im Gesundheitswesen erfreulicherweise immer weiterverbreiten und zu einer zunehmenden Formulierung von Handlungsempfehlungen führen, hinkt die praktische Umsetzung von Diagnostik und Therapie dagegen hinterher [18]. Hier werden Stimmen laut, die mehr interdisziplinäre Aus- und Weiterbildungskonzepte fordern [19, 20]. Bis diese Maßnahmen jedoch zum Tragen kommen, dauert es einige Zeit. Gegenwärtig werden die meisten ambulanten geriatrischen Patienten in erster Linie von ihren Hausärzten betreut, welche häufig aus den Fachbereichen Allgemeinmedizin oder innere Medizin kommen. Um den breiten Diagnostikbedürfnissen älterer Patienten begegnen zu können, hat die Deutsche Gesellschaft für Allgemeinmedizin und Familienmedizin 2018 die hausärztliche Leitlinie Geriatrisches Assessment in der Hausarztpraxis erarbeitet (https://www.awmf. org/uploads/tx_szleitlinien/0530151_ S1_Geriatrisches_Assessment_in_der_ Hausarztpraxis_2018-05-verlaengert. pdf.). Diese beinhaltet ein „Manageable Basis Assessment (MAGIC)“ für 
Z Gerontol Geriat 2021 ·54:255-263 https://doi.org/10.1007/s00391-020-01730-5

(c) Der/die Autor(en) 2020

\section{A. G. Barbe · S. Spiritus · A. Hagemeier · M. J. Noack · G. Röhrig}

\section{Erfassung der Mundgesundheit von ambulant betreuten Senioren durch Hausärzte. Entwicklung und Validierung des geriatrischen ambulanten Mundgesundheits-Screenings}

\section{Zusammenfassung}

Hintergrund. Einschränkungen der Mundgesundheit ambulant betreuter Senioren in Deutschland werden trotz hoher Prävalenz im hausärztlich-geriatrischen Bereich nicht routinemäßig erfasst. Da Senioren Hausärzte mit höherem Alter häufiger aufsuchen als Zahnärzte, stellt sich die Frage, ob reduzierte Mundgesundheit im Praxisalltag mit einem interdisziplinären Screeninginstrument identifiziert werden kann.

Ziel der Arbeit. Ziel der Arbeit ist, ein Screeninginstrument für reduzierte Mundgesundheit für Hausärzte zu entwickeln und dieses durch zahnmedizinische Befunde zu validieren.

Material und Methoden. Das geriatrische ambulante Mundgesundheits-Screening (GAMS) als subjektives Screeninginstrument wurde entwickelt, um für geriatrische Patienten relevante zahnmedizinische Aspekte wie Kauprobleme, Schmerzen, Parodontitis, Mundgeruch oder Mundtrockenheit in dichotomen Fragen abzubilden. Zudem erfolgt die Einschätzung der Dringlichkeit eines Zahnarztbesuches durch den Behandler. Es wurden $n=75$ Patienten eingeschlossen und der GAMS sowie eine zahnärztliche Untersuchung zur Validierung durchgeführt. Ergebnisse. Bei subjektiver Einschätzung als auch im zahnmedizinischen Befund zeigte sich reduzierte Mundgesundheit, insbesondere bei Risikofaktoren für die Entwicklung systemischer Komorbiditäten wie Dysphagie und Mangelernährung, wobei Mundgesundheitsprobleme durch die Patienten unterschätzt wurden. Einbisse,
Kauschwierigkeiten und Mundtrockenheit zeigten ausreichende Übereinstimmung zwischen oralem Befund und subjektiver Einschätzung.

Diskussion. Der GAMS könnte beitragen, die Erwägung und Berücksichtigung von Mundgesundheitsproblemen bei geriatrischen Patienten im hausärztlichen Setting zu erleichtern und die Zusammenarbeit mit Zahnmedizinern im Sinne europäischer Handlungsempfehlungen fördern.

\section{Schlüsselwörter}

Alterszahnmedizin · Kaufunktion · Mangelernährung · Geriatrische Syndrome · Mundtrockenheit

\section{Oral health assessment of seniors under outpatient care by family doctors. Development and validation of the geriatric outpatient oral health screening}

\section{Abstract}

Background. Despite a high prevalence in the general practitioner (GP) and geriatric field, limitations of oral health of seniors under outpatient care in Germany are not routinely recorded. Since seniors with a high age visit GPs more often than dentists, the question arises whether an interdisciplinary screening instrument can be used to identify reduced oral health in routine practice.

Objective. The aim of the work was to develop a screening tool for reduced oral health for GPs and to validate this by dental examinations. Material and methods. The geriatric outpatient oral health screening (GAMS) was developed as a subjective screening instrument to depict dental aspects relevant for geriatric patients, such as chewing problems, pain, periodontitis, bad breath or dry mouth in dichotomous questions. The urgency of a visit to the dentist is also assessed by the family doctor. A total of 75 patients were included and the GAMS and a dental examination were performed for validation. Results. The subjective assessment of patients and dental findings showed reduced oral health especially with recognized risk factors for the development of systemic comorbidities, such as dysphagia and malnutrition, whereas oral health problems were underestimated by patients. Bites, chewing problems and dry mouth showed sufficient correspondence between dental findings and subjective assessment. Conclusion. The GAMS could help facilitate the consideration of oral health problems in geriatric patients in a GP setting and promote cooperation with dentists in the sense of European recommendations for action.

\section{Keywords}

Geriatric dentistry · Chewing function . Malnutrition - Geriatric syndromes . Xerostomia
Hausärzte, basierend auf 9 obligat und 4 fakultativ abzufragenden Funktionsund Beschwerdebereichen. In dem fakultativen Beschwerdebereich „ungewollter Gewichtsverlust" werden mundgesundheitsbezogene Aspekte dahingehend berücksichtigt, dass Hausärzte an einen schadhaften Zahnstatus denken sollten und eine zahnärztliche Untersuchung in Erwägung ziehen sollten. Ebenso wird auf eine medikamentöse Ursache für Mundtrockenheit verwiesen und eine Liste häufig verordneter Mundtrockenheit verursachender Medikamente aufgeführt (https://www.awmf. org/uploads/tx_szleitlinien/0530151_ S1_Geriatrisches_Assessment_in_der_ Hausarztpraxis_2018-05-verlaengert. pdf). Empfehlungen über die Klärung der Frage nach Dringlichkeit einer zahnärztlichen Untersuchung finden sich in der Leitlinie jedoch ebenso wenig wie ein strukturiertes Vorgehen zur Basisdiagnostik von Mundgesundheitsproblemen.
In diesem Gesamtkontext verfolgt unsere Arbeitsgruppe in dieser Untersuchung das Ziel, die Mundgesundheit anhand eines rein subjektiven Fragebogens zu beurteilen, der den Patienten ausgehändigt werden kann und nur durch eine hausärztlich-geriatrische Einschätzung hinsichtlich der Dringlichkeit eines Zahnarztbesuches abgerundet wird, um die Hürde für Nichtzahnärzte und den zeitlichen Aufwand möglichst gering zu halten. Ein solcher Fragebogen könnte 
sowohl Patienten als auch Hausärzte für die Mundgesundheit sensibilisieren und zudem als Rechtfertigung für eine bedarfsorientierte zukünftig mögliche Überweisung zum Zahnarzt dienen.

Ziele dieser Studie waren daher die Entwicklung, Erprobung und Validierung eines subjektiven Mundgesundheitsfragebogens durch Patienten in einem ambulanten Praxissetting in Kombination mit einer Dringlichkeitseinschätzung hinsichtlich Vorstellung des $\mathrm{Pa}$ tienten beim Zahnarzt (Geriatrisches Ambulantes Mundgesundheits-Screening, GAMS).

\section{Studiendesign und Unter- suchungsmethoden}

\section{Entwicklung von Fragebogen/Einzel-}

items. In dieser Studie soll ein Erhebungsbogen für Allgemeinmediziner entwickelt werden, der die häufigsten zahnmedizinischen Probleme mit notwendigem zahnmedizinischen Handlungsbedarf bei Senioren abbildet und ohne zahnmedizinische Fachkenntnisse erhoben werden kann sowie keine klinische Munduntersuchung erfordert. Der zeitliche Aufwand sollte möglichst gering gehalten werden, um den Bogen in die tägliche Praxisroutine einbauen zu können. Dieser Fragebogen soll keine zahnmedizinische Untersuchung und Therapie ersetzen, sondern dem Hausarzt Hinweis darauf geben, ob der Patient eher zeitnah oder aber im geplanten regulären Intervall einem Zahnarzt vorgestellt werden sollte. Bei der Entwicklung des Fragebogens wird besonderes Augenmerk auf zahnmedizinische Mundgesundheitsprobleme gelegt, die in dieser Patientengruppe häufig vorkommen und zur Vermeidung von Folgestörungen einer zahnmedizinischen Therapie dienen. Hierzu wurden nach entsprechender Literaturrecherche und Definition des aktuellen Standes der Literatur [10] die folgenden Teilbereiche festgelegt ( $\bullet$ Tab. 1). Für jedes der beschriebenen zahnmedizinischen Gesundheitsprobleme wurde eine Frage entwickelt, die das beschriebene zahnmedizinische Gesundheitsproblem darstellen soll. Zudem wurde eine Gesamtfrage zur Mundgesundheit beigefügt, um überprüfen zu können, ob mit einer Frage bereits derselbe Nutzen erzielt werden könnte wie durch die individuellen themenorientierten Fragen.

Der Fragebogen wurde hinsichtlich der Formulierungen in Anlehnung an die etablierten englischsprachigen $\mathrm{BOH}-$ SE-Fragebogen [14] entwickelt, der bisher allerdings nicht auf Deutsch existiert. Zielgruppe dieser Untersuchung waren dabei nicht die geriatrischen Patienten mit akuten Zahnschmerzen, welche sich problemorientiert in die zahnärztliche Behandlung begeben. Zielgruppe waren vielmehr diejenigen geriatrischen Patienten, bei welchen das Vorliegen eines elektiven zahnmedizinischen Handlungsbedarfes überprüft werden soll. Zur möglichst einfachen und schnellen Durchführbarkeit im nichtzahnärztlichen Setting reduzierten wir den Fragenumfang auf 6 Kernfragen wurde der Fragenumfang auf 6 Kernfragen reduziert. Die Anwendbarkeit und Verständlichkeit wurden in einer Vorlaufphase von jeweils 10 Alterszahnmedizinern, 10 Hausärzten und 10 Geriatern überprüft. Dabei ergaben sich folgende Kritikpunkte:

positiv: Fragebogen ist verständlich, gut einsetzbar; der zeitliche Umfang angemessen; er ist in das geriatrische Assessment integrierbar;

negativ: Wie kann eine Vorstellung beim Zahnarzt sichergestellt werden? Was passiert mit mobilitätseingeschränkten Patienten und zahnärztlichem Handlungsbedarf?

Die Einteilung der Dringlichkeitsbewertung erfolgte empirisch auf Basis der klinischen Empfehlung der befragten Alterszahnmediziner. Hierbei wurde davon ausgegangen, dass akute Schmerzsymptomatiken im Mund von Patienten als Grund angesehen werden, zügig einen Zahnarzt aufzusuchen, und im Rahmen der Abfrage innerhalb des Assessments eher chronische Schmerzzustände adressiert werden sollen.

\section{Ein- und Ausschlusskriterien}

In diese Studie wurden nur Personen, die ambulant hausärztlich betreut werden, eingeschlossen, aufgrund des zahnärztlichen Fokus wurde „alt sein“ entsprechend den Richtlinien der Deutschen Mundge- sundheitsstudie $\mathrm{V}$ als $>75$ Jahre definiert [13]. Zusätzliche Einschlusskriterien waren die Bereitschaft, sich innerhalb einer Woche zahnärztlich untersuchen zu lassen, sowie der Ausschluss von Demenz entsprechend der vorhandenen Facharztdiagnose.

\section{Hausärztliches Vorgehen}

Den 75 eingeschlossenen, ambulant betreuten Patienten wurde der Fragebogen zur Beantwortung im Wartezimmer gegeben. Im Einzelfall wurden die Patienten durch medizinisches Assistenzpersonal beim Ausfüllen unterstützt, wobei die Vorstellung in der hausärztlichen Praxis im Rahmen eines medizinisch indizierten Besuches erfolgte. Alter, BMI, Komorbiditäten und ggf. aktualisierter Medikationsplan konnten aus der Krankenakte entnommen werden. Die Diagnose eines Diabetes mellitus wurde im Vorfeld gestellt, wenn in der laborchemischen Untersuchung der Nüchternwert für Glucose mindestens einmalig einen Wert über $126 \mathrm{mg} / \mathrm{dl}$ gezeigt hatte oder der $\mathrm{HbA}_{1 c}$-Wert $\geq 6,5 \%$ war. Hierbei ist kritisch anzumerken, dass es im Falle einer späteren Unterschreitung der Werte nicht zu einer Rückcodierung der Variable Diabetes kam, sodass möglicherweise überpositive Prävalenzen berichtet wurden. Die Durchsicht des bereits vorausgefüllten Bogens wurde im Behandlungszimmer durch den Hausarzt durchgeführt, und anhand der gegebenen Patientenantworten wurde die Dringlichkeit eines Zahnarztbesuches folgendermaßen bewertet:

1. Der Patient kreuzt nur „nein“ angekreuzt und ein Zahnarztbesuch innerhalb der letzten 6 Monate: kein Grund zur Überweisung zum Zahnarzt.

2. Der Patient kreuzt ein- bis 2-mal „ja“ angekreuzt und ein Zahnarztbesuch liegt länger als 3 Monate zurück: es besteht Grund zur Überweisung zum Zahnarzt in 2 Monaten.

3. Der Patient kreuzt 3- bis 4-mal ,ja“ angekreuzt: der Patient sollte in den nächsten 2 Wochen einen Termin beim Zahnarzt vereinbaren.

4. Der Patient kreuzt mehr als 4-mal ,ja“ angekreuzt oder akute Schmerzen: 


\section{Tab. 2 Klinische Charakteristika}

\begin{tabular}{l|l}
\hline & Gesamt ( $\mathbf{n = 7 5 )}$ \\
\hline Geschlecht (weiblich) & $45(60)$ \\
\hline Alter in Jahren & $80 \pm 4$ \\
\hline Größe in cm & $168 \pm 7$ \\
\hline Gewicht in kg & $76 \pm 13$ \\
\hline BMI & $27 \pm 4$ \\
\hline Antiphlogistikaeinnahme & $31(82)$ \\
\hline Rheumatoide Arthritis & $4(10)$ \\
\hline Diabetes Typ 2 & $29(82)$ \\
\hline Hauszahnarzt vorhanden & $66(88)$ \\
\hline Polypharmazie (>5) & $39(52)$ \\
\hline Anzahl der Diagnosen, Komorbiditäten & $9(7 ; 14)$ \\
\hline Anzahl der Medikamente & $6 \pm 4$ \\
\hline Kategoriale Merkmale werden als abs. Häufigkeiten mit Prozent $n$ & $(\%)$, normal verteilte stetige \\
\hline Merkmale als Mittelwert \pm Standardabweichung, nichtnormal verteilte Merkmale als Median mit \\
\hline Interquartilsabstand (IQRo,25; IQRo,75) angegeben \\
\hline
\end{tabular}

sofortiger Termin beim Zahnarzt empfohlen.

Da weder eine formgerechte noch eine formlose Überweisung vom Hausarzt zum Zahnarzt aktuell berufsrechtlich zulässig ist, wurde dem Patienten ausschließlich eine mündliche Empfehlung, sich zahnärztlich vorzustellen, ausgesprochen.

\section{Zahnmedizinische Untersuchung}

Die Validierung des Instrumentes erfolgte in einem weiteren Schritt durch eine zahnärztliche Untersuchung und Überprüfung der hausärztlichen Dringlichkeitseinschätzung. Die subjektiven Einzelitems sowie die zusammenfassende Einzelfrage sollten hierbei verglichen werden, um die mögliche Übereinstimmungen zu dokumentieren. Die zahnmedizinischen Untersuchungsbefunde als objektive Parameter wurden mit der subjektiven Einschätzung der Patienten (- Tab. 1) verglichen. Eine Darstellung der zahnmedizinischen Parameter erfolgt im Zusatzmaterial online: „Zahnmedizinische Untersuchungsbefunde“.

\section{Statistische Auswertung}

Nach der Datenaufbereitung erfolgte eine deskriptive Analyse zur Beschreibung des Kollektivs. Dabei wurden stetige

\section{Ethikvotum}

Die Studie wurde unter der Nummer 18-324 durch die Ethikkommission der Universität Köln positiv bewertet. Eine Studienregistrierung beim DRKS (www. drks.de) erfolgte unter der Nummer DRKS00015630. Die Studie wurde entsprechend der Deklaration von Helsinki/ Good clinical practice durchgeführt.

\section{Ergebnisse}

Klinische Charakteristika. Die Studienteilnehmer waren zu $60 \%$ weiblich, $40 \%$ männlich. Die Teilnehmer zeigten eine hohe Prävalenz der Einnahme von Antiphlogistika (82\%) und Diabetes mellitus Typ 2 (82\%). Mittleres Alter war 80 (SD 4), im Mittel wurden 10 (SD 5) Komorbiditäten angegeben und 6 (SD 4) Medikamente täglich eingenommen (- Tab. 2). Der letzte Zahnarztbesuch lag im Mittel über ein Jahr zurück.

Geriatrisches ambulantes Mundgesundheits-Screening. Hinsichtlich der eigenen subjektiven Einschätzung ihrer Mundgesundheit zeigten sich insbesondere bei den Teilbereichen „Probleme beim Kauen oder Schlucken“ (19\%), „Mundgeruch“ (12\%), „Mundtrockenheit“ (40\%) sowie dem Teilbereich „Vorhandensein von Prothesen" (73\%) auffällige Befunde. Die Beantwortung der Frage zu Problemen hinsichtlich der Gesamtmundgesundheit wurde von $15 \%$ der Studienteilnehmer bejaht (• Tab. 3). Wenn die Einzelitems anhand von Kreuztabellen in Relation zu der Gesamtfrage „Probleme im Mundbereich“ gestellt wurden, bestand bei folgenden Items eine gute Übereinstimmung $(<5 \%$-Angabe eines Problems beim Einzelitem, aber keine Bejahung des Gesamtitems „Probleme im Mundbereich“), durch die Patienten: „Missempfindung/Brennen“ (3\%), „Schmerzen im Mund“ (3\%), „Zahnfleischbluten“ (4,7\%).

Dahingegen bestand eine geringere Übereinstimmung bei der subjektiven Bewertung der „Probleme beim Kauen und Schlucken“ (17\%), „Mundge-

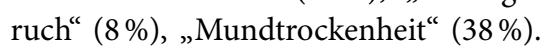
Das bedeutet, dass bei Vorliegen dieser Probleme diese nicht als Problem im 
Tab. 3 Zahnärztlicher Untersuchungsbefund

\begin{tabular}{|c|c|}
\hline & Gesamt $(n=75)$ \\
\hline Prothesendruckstellen & $14(19)$ \\
\hline Rhagaden & $12(16)$ \\
\hline Visuelle Candida-Besiedlung & $2(3)$ \\
\hline Hyposalivation, unstimuliert ( $<0,2 \mathrm{ml} / \mathrm{min})$ & $14(19)$ \\
\hline Hyposalivation, stimuliert (<0,7 ml/min) & $14(19)$ \\
\hline Halitosis (Organoleptischer Score $>0$ ) [22] & $57(76)$ \\
\hline API & $50(24 ; 100)$ \\
\hline SBI & $11(0 ; 37)$ \\
\hline $\mathrm{DHI}$ & $2(0 ; 8)$ \\
\hline Zahnzahl & $14 \pm 10$ \\
\hline Ersetzte Zähne & $9(0 ; 25)$ \\
\hline$P S I_{\max }$ & $2,7 \pm 0,9$ \\
\hline$P S I_{\text {mean }}$ & $2,3 \pm 0,7$ \\
\hline \multicolumn{2}{|c|}{$\begin{array}{l}\text { API approximaler Plaque-Index, SBI Sulcus Bleeding Index, DHI Denture Hygiene Index } \\
\text { Kategoriale Merkmale werden als abs. Häufigkeiten mit Prozent } n(\%) \text {, normal verteilte stetige } \\
\text { Merkmale als Mittelwert } \pm \text { Standardabweichung, nichtnormal verteilte Merkmale als Median mit } \\
\text { Interquartilsabstand }\left(\mathrm{IQR}_{0,25} ; \mathrm{IQR} 0,75\right) \text { angegeben }\end{array}$} \\
\hline
\end{tabular}

Mundbereich abgebildet wurden. Zudem wurde eine Variable erstellt, bei der die Einzelitems als Mundprobleme zusammengefasst wurden ( $\mathrm{ja}$, wenn mindestens eines der Probleme, und nein, wenn keines der Probleme angegeben wurde). 53 Patienten (83\%) haben die globale Frage mit nein beantwortet, obwohl sie mindestens eines der Einzelprobleme mit ja beantwortet haben.

Zahnmedizinischer Befund. Im zahnärztlichen Untersuchungsbefund waren folgende Befunde auffällig (• Tab. 3): Druckstellen unter Prothesen, Rhagaden, Speichelmangel. Die Studienteilnehmer zeigten parodontalen Behandlungsbedarf, eine mittlere Zahnzahl von 14 (SD 10) und eine mangelnde Prothesenhygiene.

\section{Dringlichkeitseinschätzung des Zahn-} arztbesuches. Während der umgehende Zahnarztbesuch vom Hausarzt bei $1 \%$ [1] der Studienteilnehmer angenommen wurde, war dieser bei 3\% [2] bei der Untersuchung durch den Zahnarzt indiziert. Die Vorstellung in den nächsten 2 Wochen wurde durch den Hausarzt in $9 \%$ [7] der Fälle vs. $28 \%$ [17] der Fälle durch den Zahnarzt bewertet und die Vorstellung beim Zahnarzt innerhalb der nächsten 2 Monate bei $63 \%$ [N=47] bewertet durch den Hausarzt vs. $56 \%$
[N=42] durch den Zahnarzt. Bezüglich der notwendigen sofortigen Vorstellung beim Zahnarzt aus Hausarztsicht wurde dies bei $3 \%$ durch den Zahnarzt als nicht so dringlich angesehen; bei der Vorstellung innerhalb der nächsten 2 Wochen erfolgte in $24 \%$ der Fälle eine Unterschätzung durch den Hausarzt und bei der Vorstellung innerhalb der nächsten 2 Monate eine $43 \%$ ige differente Einschätzung. Zusammenfassend wurde die Notwendigkeit eines Zahnarztbesuches durch den Zahnarzt als dringlicher eingeschätzt als durch den Hausarzt.

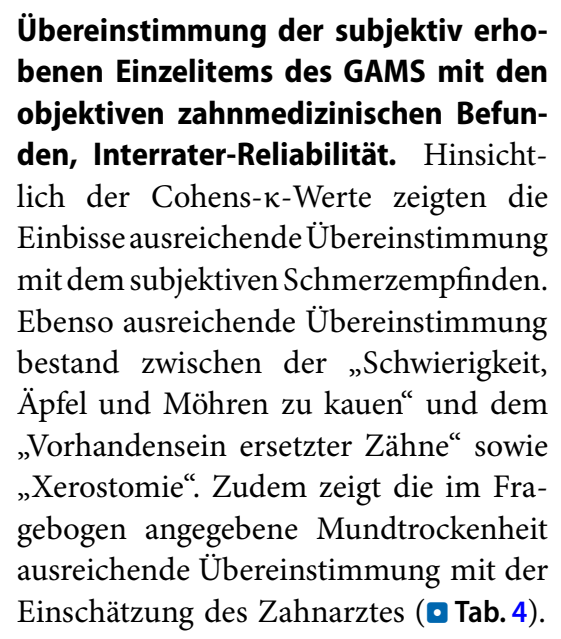

\section{Diskussion}

In diesem Projekt wurden die Entwicklung und Validierung des GAMS zur Erfassung der Mundgesundheit von ambulant betreuten Senioren durch Hausärzte und Geriater beschrieben. Hierbei wurden in der Gruppe der Senioren hochprävalente zahnmedizinische Teilbereiche definiert und diesen Teilbereichen einfache für Patienten im täglichen Praxisablauf in einer Hausarztpraxis beantwortbare binäre Fragen zugeordnet. $\mathrm{Zu}$ dem wurde eine Frage zur Gesamtmundgesundheit entwickelt und hinsichtlich ihrer Aussagekraft überprüft. Es erfolgte zusätzlich die zahnmedizinische Evaluation der Mundgesundheit und somit Validierung der subjektiven genannten Antworten.

Die sowohl bei der subjektiven Einschätzung der Patienten als auch bei der zahnmedizinischen Untersuchung diagnostizierten Mundgesundheitseinschränkungen wie „Probleme beim Kauen oder beim Schlucken“, „Mundgeruch“ und „Mundtrockenheit“ weisen auf die im geriatrischen Kollektiv besonders häufig auftretenden Mundgesundheitsprobleme hin, welche inzwischen anerkannte Risikofaktoren für die Entwicklung systemischer Komorbiditäten und Folgestörungen sind, wie Dysphagie, Mangelernährung oder Pneumonie [23-25]. Der von uns entwickelte GAMS könnte hier ansetzen und dazu beitragen, die Erwägung und Berücksichtigung von Mundgesundheitsproblemen bei ambulanten geriatrischen Patienten im hausärztlichen Setting zu erleichtern und die Zusammenarbeit mit Zahnmedizinern im Sinne europäischer Handlungsempfehlungen $\mathrm{zu}$ fördern. Zur optimalen Betreuung multimorbider geriatrischer Patienten ist ein ganzheitlicher Diagnostik- und Therapieansatz auf der Basis des multidimensionalen geriatrischen Assessments notwendig. Die interdisziplinäre Zusammenarbeit mit Fachgruppen wie Ergotherapie, Physiotherapie, Logopädie und Neuropsychologie wird heute sogar durch die Abrechnungsmodalitäten der geriatrischen Komplexbehandlung berücksichtigt. Inzwischen gibt es auch zertifizierte Kooperationszentren zwischen 
Tab. 4 Cohens $\mathrm{k}$ für die subjektiven Einzelitems des geriatrischen ambulanten Mundgesundheits-Screenings mit den objektiven zahnmedizinischen Befunden

\begin{tabular}{|c|c|c|}
\hline $\begin{array}{l}\text { Subjektive Einschätzung } \\
n(\%)\end{array}$ & $\begin{array}{l}\text { Objektiver zahnme- } \\
\text { dizinischer Befund }\end{array}$ & $\begin{array}{l}\text { Cohens } \\
\mathbf{K}\end{array}$ \\
\hline \multirow{4}{*}{$\begin{array}{l}\text { Leiden Sie an Missempfindungen wie Brennen oder } \\
\text { pelzigem Gefühl im Mund? } \\
3 \text { (4) }\end{array}$} & Druckstellen & $-0,071$ \\
\hline & Einbisse & $-0,053$ \\
\hline & Candidiasis & $-0,033$ \\
\hline & Rhagaden & 0,074 \\
\hline \multirow{6}{*}{$\begin{array}{l}\text { Haben Sie Schmerzen oder andere Beschwerden im Bereich } \\
\text { von Mund und Zähnen? } \\
7 \text { (9) }\end{array}$} & Druckstellen & $-0,033$ \\
\hline & Einbisse & 0,277 \\
\hline & Candidiasis & $-0,043$ \\
\hline & Rhagaden & 0,105 \\
\hline & Kariöse Zähne & 0,075 \\
\hline & Zerstörte Zähne & $-0,043$ \\
\hline \multirow{4}{*}{$\begin{array}{l}\text { Haben Sie Zahnfleischbluten beim Putzen, beim Essen oder } \\
\text { auch spontan? } \\
6 \text { (8) }\end{array}$} & $\begin{array}{l}\text { Approximaler } \\
\text { Plaque-Index }\end{array}$ & \\
\hline & Sulcus-Blutungsindex & \\
\hline & Gesamt-PSI & \\
\hline & $\begin{array}{l}\text { Mittlerer höchster } \\
\text { PSI-Wert }\end{array}$ & $\begin{array}{l}0,044 \\
0,033\end{array}$ \\
\hline \multirow{7}{*}{$\begin{array}{l}\text { Haben Sie Schwierigkeiten beim Zerkauen und beim } \\
\text { Schlucken von Möhren und Äpfeln? } \\
14 \text { (19) }\end{array}$} & Zahnzahl & - \\
\hline & Ersetzte Zähne & 0,217 \\
\hline & Kariöse Zähne & 0,034 \\
\hline & $\begin{array}{l}\text { Unstimulierte Spei- } \\
\text { chelfließrate }\end{array}$ & 0,122 \\
\hline & $\begin{array}{l}\text { Stimulierte Speichel- } \\
\text { fließrate }\end{array}$ & 0,122 \\
\hline & Xerostomie & 0,208 \\
\hline & Totalprothese & - \\
\hline $\begin{array}{l}\text { Ist Ihnen selbst ein unangenehmer Geruch aus dem Mund } \\
\text { aufgefallen, oder hat Sie jemand darauf angesprochen? } \\
9(12)\end{array}$ & $\begin{array}{l}\text { Organoleptischer } \\
\text { Index }(0-3)\end{array}$ & 0,083 \\
\hline \multirow{3}{*}{$\begin{array}{l}\text { Haben Sie öfter das Gefühl, dass Ihr Mund trocken ist? } \\
30 \text { (40) }\end{array}$} & Xerostomie & 0,318 \\
\hline & $\begin{array}{l}\text { Stimulierte Speichel- } \\
\text { fließrate }\end{array}$ & 0,085 \\
\hline & $\begin{array}{l}\text { Unstimulierte Spei- } \\
\text { chelfließrate }\end{array}$ & 0,146 \\
\hline
\end{tabular}

Fett gedruckte Werte repräsentieren statistisch signifikante Korrelationen $(p<0,05)$.

Geriatern und Alterstraumatologen. Kooperationen zwischen Hausärzten und Fachärzten werden im Sinne einer guten Patientenversorgung im Vertragsarztänderungsgesetz [26], das seit 2007 in Kraft ist, sogar ausdrücklich gefördert, sei es in Form von Berufsausübungsgemeinschaften oder innerhalb medizinischer Versorgungszentren (MVZ). Bedauerlicherweise sind jedoch Kooperationen zwischen Vertragshausärzten und Vertragszahnärzten davon ausgeschlossen; eine Praxisgemeinschaft oder eine Berufsausübungsgemeinschaft ist nicht möglich. Auch die noch immer weitgehend getrennte Ausbildung von Human- und Zahnmedizinstudenten verhindert eine gerade im geriatrischen Setting dringend notwendige spätere $\mathrm{Zu}$ sammenarbeit aufgrund unzureichender Fachkenntnisse und eines dadurch oft eingeschränkten Stellenwerts in der Beurteilung des jeweils anderen Fachbereiches. Aufgrund der hohen Relevanz mundgesundheitsbezogener Defizite mit Einfluss auf die Allgemeingesundheit geriatrischer Patienten sind feste interdisziplinäre Versorgungsstrukturen nicht nur wünschenswert, sondern für eine optimale Betreuung auch essenziell.
Bis die entsprechenden kooperationserleichternden Strukturen geschaffen sind, können Screeningbogen wie der GAMS zu einer Sensibilisierung für mundgesundheitsbezogene Probleme unter Haus- und Fachärzten beitragen und frühzeitig auf Defizite aufmerksam machen. Die Kontaktaufnahme zum Zahnarzt muss vorerst aufgrund fehlender Strukturvorgaben über persönliche Netzwerkbildung laufen, ergänzt durch datenschutzwahrende erweiterte Kommunikation (z.B. durch das Versenden von Befunden).

Ein mundgesundheitsbezogenes Screening im hausärztlichen Setting kann zudem zu einer Verbesserung der Patientenzufriedenheit beitragen. Eine Freiburger Literaturanalyse ergab 2016, dass interprofessionelle Zusammenarbeit nicht nur zu einer größeren Zufriedenheit zwischen den Professionen beitrug, sondern auch zu einer verbesserten Patientenzufriedenheit führte [27]. So kann eine hausärztliche Frage zur prothetischen Versorgung bereits auf ein Versorgungsdefizit und einen Versorgungsbedarf unter älteren Patienten hinweisen, wie die Ergebnisse unserer Untersuchung ergaben:

Unsere subjektive Befragung hinsichtlich vorhandener Prothesen ergab eine hohe Prävalenz. Basierend auf unseren klinischen Erfahrungen ist hierbei problematisch, dass viele Patienten das Vorhandensein von Prothesen nicht mit einer notwendigen regelmäßigen Kontrolle beim Zahnarzt assoziieren, während der Hausarzt durchaus weiteraufgesucht wird. Eine regelmäßige Nachsorge hinsichtlich Funktionalität, Passform, hygienischer Situation ist notwendig, wird aber von den Patienten häufig vernachlässigt. Hier kann eine Abfrage allein der Prävalenz und der damit verbundenen Aufforderung, einen Zahnarzt regelmäßig aufzusuchen, helfen, mögliche Probleme frühzeitig zu identifizieren und zu beheben.

Unser auf interprofessionelle Zusammenarbeit ausgerichteter Screeningbogen GAMS würde das Spektrum der bereits existierenden interdisziplinären Assessments erweitern: So sind beispielweise Assessmentinstrumente für ein Diabetesscreening, die in der Zahn- 
arztpraxis angewendet werden können, beschrieben worden (FINDRISK) [28]. Der GAMS mag ein entsprechender erster Schritt zu einem Assessmentinstrument zur Vorstellung bei Zahnarzt sein. Dafür müssten aber, wie bereits erwähnt, die aktuellen berufsrechtlichen Rahmenbedingungen angepasst werden, damit eine Überweisung vom Hausarzt zum Zahnarzt zulässig wird. Die durch uns etablierte Gesamtfrage nach Problemen im Mundbereich bildet die Häufigkeit der Beschwerden, erfragt durch Einzelitems, nicht ab, sodass eine differenziertere Abfrage notwendig erscheint. Wie in der Literatur beschrieben, werden einzelne der in den Einzelitems abgefragten Probleme von Patienten nicht als mundgesundheitsrelevante Probleme eingeschätzt, und diese werden häufig nicht berichtet. Daher schätzen wir die individuelle Abfrage als zielführender ein als eine Gesamtfrage, die die hohe Prävalenz von Mundgesundheitsproblemen nicht abbildet.

Hinsichtlich der Dringlichkeit wurde die Notwendigkeit einer zahnärztlichen Vorstellung leicht zeitkritischer eingeschätzt als durch den Hausarzt, in den meisten Fällen war die Einschätzung aber identisch. Natürlich wäre die korrekte Einordnung der Dringlichkeit auf hausärztlicher Seite wünschenswert, da dadurch eine Gefahr der Unterbehandlung vermieden werden könnte. Dennoch entsteht aufseiten der Hausärzte keine Verantwortlichkeit zu einer korrekten Einschätzung bzw. keine Verantwortlichkeit zu einer Überweisung. Der Bogen soll ausschließlich einer Unterstützung dienen und im hausärztlichen Bereich bei der Betreuung geriatrischer Patienten für Mundgesundheitsprobleme sensibilisieren. Es kann daher diskutiert werden, ob nicht auch eine reine Abfrage der Zeitspanne seit dem letzten Zahnarztbesuch des Patienten gereicht hätte, um eine Vorstellung zu rechtfertigen. Dennoch erscheint die hohe Relevanz von Mundgesundheitsproblemen mit Einfluss auf die Allgemeingesundheit im höherem Alter als Rechtfertigung dafür, diese auch differenziert abzufragen und in die hausärztliche Therapieplanung einzubauen.

Hinsichtlich der Interrater-Reliabilität zwischen Zahnarzt und der subjekti- ven Einschätzung kann ebenfalls nur bei den relevanten Mundgesundheitsproblemen subjektives Schmerzempfinden, Kauschwierigkeiten, ersetzte Zähnen sowie Mundtrockenheitsproblematik ausreichende Übereinstimmung gezeigt werden. Dies mag ebenfalls zum einen an der Unterschätzung der Mundgesundheitsprobleme der Patienten im Kontext einer reduzierten Allgemeingesundheit mit anderen relevanten und stärker subjektiv beeinträchtigenden Gesundheitsproblemen liegen. Zum anderen kann es aber auch durch die relativ kleinen Stichprobengrößen bedingt sein, denn bei höheren Prävalenzen $(n>30)$ wurden die $\kappa$-Übereinstimmungswerte deutlicher.

Grundsätzlich müssen in der Diskussion zudem diejenigen Aspekte der Versorgung, die über die direkte Versorgung durch Zahn- und Hausärzte hinausgehen, sich aber dennoch in mangelnder Mundgesundheit ausdrücken, wie etwa ungedeckte Pflege- und Rehabilitationsbedarfe, berücksichtigt werden. Ganz grundsätzlich sind Senioren mit zunehmender Pflegebedürftigkeit weniger belastbar: Therapiefähigkeit, Mundhygienefähigkeit und Eigenverantwortung nehmen ab, und ein Drittel der Menschen mit Pflegebedarf kann sich nicht mehr eigenständig um die Mund- und Prothesenhygiene kümmern und benötigt Hilfe [13]. Multiple systemische Erkrankungen, Multimorbidität, Demenz, reduzierte Mobilität und häufige Polypharmazie verschlechtern und potenzieren die Situation zusätzlich und hindern sie selbst und das Pflegepersonal zunehmend, gute Mundhygienemaßnahmen durchzuführen [17, 18]. So stellt allein die Tatsache, dass jemand für Mundhygienemanöver externe Hilfe benötigt, ein erhöhtes Risiko für eine reduzierte Mundgesundheit dar. Die daher geforderte Unterstützung in späteren Lebensphasen bei der Mundpflege durch das Pflegepersonal soll die reduzierte Mundhygienefähigkeit der Patienten ersetzen. Ob dies bei den beschriebenen Risikofaktoren selbst bei perfekter Durchführung zum Erhalt guter Mundgesundheit ausreichen kann, bleibt fraglich. Zudem werden in der Literatur aufseiten der Pflegekräfte or- ganisatorische Missstände angegeben: zu wenig Zeit, häufige Personalwechsel, aber auch wenig eigenes Gesundheitsbewusstsein im Hinblick auf die eigene Mundhöhle. Häufig wird mangelnde Ausbildung der Pflegekräfte in Bezug auf Mundhygieneprävention im Heim genannt, allerdings auch fehlende zahnärztliche Ansprechpartner sowie der Mangel an wirksamen Strategien, dem Unwillen zur Mitarbeit bei den Bewohnern zu begegnen [19, 20, 29, 30].

\section{Limitationen}

Da diese Pilotuntersuchung zunächst als monozentrische Untersuchung in einer Praxis durch einen Hausarzt und durch eine Zahnärztin überprüft wurde, in nur einem schon langfristig kontinuierlich angebundenen Patientenkollektiv, ist die externe Validität der Daten begrenzt. Eine Validierung des Fragebogens mit multizentrischem Einsatz und höheren Patientenzahlen sollen der Inhalt einer Folgeuntersuchung werden. Ebenso stellt sich hinsichtlich der praktischen Umsetzung die Frage, ob vonseiten der Hausärzte ein solches Instrument praktikabel erscheint und in welcher Frequenz dieses angewandt werden müsste, um wirksam zu sein. Auch dies sollte Teil weiterführender Untersuchungen sein.

\section{Fazit für die Praxis}

Der Einsatz eines Assessmentinstruments zur subjektiven Mundgesundheit zum Einsatz in der hausärztlichen Routine, das relevante und hochprävalente Mundgesundheitsparameter bei Senioren und geriatrischen Patienten abbildet, kann Hausärzten, Geriatern und Patienten helfen, Mundgesundheitsprobleme zu identifizieren. Da der Hausarzt auch für hochaltrige Patienten gewöhnlich der erste Ansprechpartner bleibt, diese Patienten aber aus der zahnärztlichen Versorgung immer stärker herausfallen, kann durch ein solches Assessmentinstrument zudem die Patientensensibilität für Mundgesundheit erhöht werden, bis hin zu regelmäßigen kontroll- und präventionsorientierten Terminen. Entsprechend den europäischen Forderungen sollte bei der Be- 
handlung geriatrischer Patienten angestrebt werden, die strikte Trennung der Fächer Zahnmedizin und Medizin zu überwinden, um im Sinne der Patienten eine interdisziplinäre Betreuung zumindest von sich beidseitig beeinflussenden Gesundheitsproblemen zu ermöglichen.

\section{Korrespondenzadresse}

PD Dr. Dr. Anna Greta Barbe
Fachbereich Parodontologie,
AG Seniorenzahnmedizin,
Poliklinik für Zahnerhaltung
u. Parodontologie, Uniklinik
Köln
Kerpener Str. 32, 50931 Köln,
Deutschland
anna.barbe@uk-koeln.de

Funding. Open Access funding provided by Projekt DEAL.

\section{Einhaltung ethischer Richtlinien}

Interessenkonflikt. A.G. Barbe, S. Spiritus, A. Hagemeier, M.J. Noack und G. Röhrig geben an, dass kein Interessenkonflikt besteht.

Die Studie wurde unter der Nummer 18-324 durch die Ethikkommission der Universität Köln positiv bewertet. Eine Studienregistrierung beim DRKS (www.drks. de) erfolgte unter der Nummer DRKS00015630. Die Studie wurde entsprechend der Deklaration von $\mathrm{Hel}-$ sinki/Good clinical practice durchgeführt. Von allen beteiligten Patienten liegt eine Einverständniserklärung vor.

Open Access. Dieser Artikel wird unter der Creative Commons Namensnennung 4.0 International Lizenz veröffentlicht, welche die Nutzung, Vervielfältigung, Bearbeitung, Verbreitung und Wiedergabe in jeglichem Medium und Format erlaubt, sofern Sie den/die ursprünglichen Autor(en) und die Quelle ordnungsgemäß nennen, einen Link zur Creative Commons Lizenz beifügen und angeben, ob Änderungen vorgenommen wurden.

Die in diesem Artikel enthaltenen Bilder und sonstiges Drittmaterial unterliegen ebenfalls der genannten Creative Commons Lizenz, sofern sich aus der Abbildungslegende nichts anderes ergibt. Sofern das betreffende Material nicht unter der genannten Creative Commons Lizenz steht und die betreffende Handlung nicht nach gesetzlichen Vorschriften erlaubt ist, ist für die oben aufgeführten Weiterverwendungen des $\mathrm{Ma}$ terials die Einwilligung des jeweiligen Rechteinhabers einzuholen.

Weitere Details zur Lizenz entnehmen Sie bitte der Lizenzinformation auf http://creativecommons.org/ licenses/by/4.0/deed.de.

\section{Literatur}

1. Barbe AG, Bock N, Derman SH, Felsch $M$, Timmermann L, Noack MJ (2017) Self-assessmen of oral health, dental health care and oral healthrelated quality of life among Parkinson's disease patients. Gerodontology 34(1):135-143

2. Barbe AG, Schmidt $P$, Bussmann $M$, Kunter $H$, Noack MJ, Rohrig G (2018) Xerostomia and hyposalivation in orthogeriatric patients with fall history and impact on oral health-related quality of life. Clin Interv Aging 13:1971-1979

3. Semba RD, Blaum CS, Bartali B, Xue QL, Ricks MO, Guralnik JM et al (2006) Denture use, malnutrition frailty, and mortality among older women living in the community. J Nutr Health Aging 10(2):161-167

4. Samnieng P, Ueno M, Shinada K, ZaitsuT, WrightFA, Kawaguchi Y (2012) Association of hyposalivation with oral function, nutrition and oral health in community-dwelling elderly Thai. Community Dent Health 29(1):117-123

5. Torres MJ, Feart C, Samieri C, Dorigny B, Luiking Y, Berr $C$ et al (2015) Poor nutritional status is associated with a higher risk of falling and fracture in elderly people living at home in France: the three-city cohort study. Osteoporos Int 26(8):2157-2164

6. Michel A, Verin E, Gbaguidi X, Druesne L, Roca F, Chassagne P (2018) Oropharyngeal dysphagia in community-dwelling older patients with dementia: prevalence and relationship with geriatric parameters. J Am Med Dir Assoc 19(9):770-774

7. Melgaard D, Rodrigo-Domingo $M$, Morch MM (2018) The prevalence of oropharyngeal dysphagia in acute geriatric patients. Geriatrics (Basel) 3(2):E15. https://doi.org/10.3390/ geriatrics3020015

8. Gingrich A, Volkert D, Kiesswetter E, Thomanek M Bach S, Sieber CC et al (2019) Prevalence and overlap of sarcopenia, frailty, cachexia and malnutrition in older medical inpatients. BMC Geriatr 19(1):120

9. Barbe AG (2018) Medication-induced xerostomia and hyposalivation in the elderly: culprits, complications, and management. Drugs Aging 35(10):877-885. https://doi.org/10.1007/s40266 018-0588-5

10. Astvaldsdottir A, Bostrom AM, Davidson T, Gabre P, Gahnberg L, Sandborgh Englund G et al (2018) Oral health and dental care of older persons-a systematic map of systematic reviews. Gerodontology 35(4):290-304

11. van derPutten GJ, de BaatC, De Visschere L, Schols J (2014) Poor oral health, a potential new geriatric syndrome. Gerodontology 31(1):17-24

12. Priyadarshini SR, Sahoo PK, Bhuyan SK, Misra SR, Pati AR (2014) Growing old is mandatory but growing up is optional: an explanation to geriatrics. J Clin Diagn Res 8(12):ZE22-4

13. Jordan RA, Bodechtel C, Hertrampf K, Hoffmann T, Kocher T, Nitschke I et al (2014) The fifth German oral health study (Fünfte deutsche Mundgesundheitsstudie, DMS V)—rationale, design, and methods. BMC Oral Health 14:161

14. Kayser-Jones J, Bird WF, Paul SM, Long L, Schell ES (1995) An instrument to assess the oral health status of nursing home residents. Gerontologist 35(6):814-824

15. Chalmers JM, King PL, Spencer AJ, Wright FA, Carter KD (2005) The oral health assessment tool-validity and reliability. Aust Dent J 50(3):191-199
16. Wostmann B, Seelbach M, Seelbach P, Podhorsky A Kolb GF, Bretzel RG et al (2017) Mini dental assessment: a simple screening test for non-dental staff.Clin Oral Investig 21(5):1457-1464

17. Nitschke I, llgner A, Müller F (2005) Barriers to provision of dental care in long-term care facilities: the confrontation with ageing and death. Gerodontology 22(3):123-129

18. Zenthofer A, Baumgart D, Cabrera T, Rammelsberg P, Schroder J, Corcodel N et al (2017) Poor dental hygiene and periodontal health in nursing home residents with dementia: an observational study. Odontology 105(2):208-213

19. Hoad-Reddick G, Grant AA, Griffiths CS (1990) Investigation into the cleanliness of dentures in an elderly population. J Prosthet Dent 64(1):48-52

20. Adams R (1996) Qualified nurses lack adequate knowledge related to oral health, resulting in inadequate oral care of patients on medical wards. JAdv Nurs 24(3):552-560

21. Landis JR, Koch GG (1977) The measurement of observer agreement for categorical data. Biometrics 33(1)159-174. https://doi.org/10. $2307 / 2529310$

22. Tonzetich J (1977) Production and origin of oral malodor: a review of mechanisms and methods of analysis. J Periodontol 48:13-20

23. Gil-Montoya JA, de Mello AL, Barrios R, GonzalezMoles MA, Bravo M (2015) Oral health in the elderly patient and its impact on general wellbeing: a nonsystematic review. Clin Interv Aging 10:461-467

24. Tashiro H, Kikutani T, Tamura F, Takahashi N, Tohara T, Nawachi K et al (2019) Relationship between oral environment and development of pneumonia and acute viral respiratory infection in dependent older individuals. Geriatr Gerontol Int 19(11):1136-1140

25. Kikutani T, Tamura F, Tashiro $H$, Yoshida $M$, Konishi K, Hamada R (2015) Relationship between oral bacteria count and pneumonia onset in elderly nursing home residents. Geriatr Gerontol Int 15(4):417-421

26. https://www.aerzteblatt.de/archiv/55669/ Vertragsarztrechtsaenderungsgesetz-(II)-NeueFreiheiten-erfordern-Visionen. Zugegriffen: 20.04.2020

27. Korner M, Butof S, Muller C, Zimmermann L, Becker S, Bengel J (2016) Interprofessional teamwork and team interventions in chronic care: a systematic review. J Interprof Care 30(1):15-28

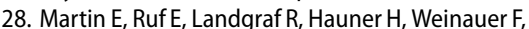
Martin S (2011) FINDRISK questionnaire combined with $\mathrm{HbA} 1 \mathrm{c}$ testing as a potential screening strategy for undiagnosed diabetes in a healthy population. Horm Metab Res 43(11):782-787. https://doi.org/10.1055/s-0031-1286333

29. Paley GA, Slack-Smith LM, O'Grady MJ (2004) Aged care staff perspectives on oral care for residents: Western Australia. Gerodontology 21(3):146-154

30. Hoben M, Clarke A, Huynh KT, Kobagi N, Kent A, $\mathrm{Hu} \mathrm{H}$ et al (2017) Barriers and facilitators in providing oral care to nursing home residents, from the perspective of care aides: a systematic review and meta-analysis. Int J Nurs Stud 73:34-51 